\title{
Dynamics of the Labyrinthine Patterns at the Diffuse Phase Boundaries
}

\author{
A. Cēbers \\ Institute of Physics, University of Latvia, Salaspils-1, LV-2169, Latvia
}

Received on 15 February, 2001

\begin{abstract}
The phase diagram of a magnetic colloid in a Hele-Shaw cell is calculated. As a function of the magnetic field strength, of the concentration and of the layer thickness the magnetic colloid can find itself in a stripe phase, the hexagonal phase or in an unmodulated state. Those results allow to interpret experiments observing the transformation of a labyrinthine pattern into a hexagonal structure. This possibility is confirmed directly by the numerical simulation presented here and showing the transformation of the labyrinthine pattern into the hexagonal structure.
\end{abstract}

\section{Introduction}

The wonderful labyrinthine patterns formed by the magnetic liquids in the Hele-Shaw cells [1] have drawn attention from different points of view [2]. Investigations carried out so far show that the labyrinthine patterns formed by the monophasic liquids conserves their topology and does not break up into a set of separate droplets $[1,3,4]$ as could be expected due to the mutual repulsion of the dipoles. Although it is illustrated in the frame of the linear theory that the magnetic liquid stripes are unstable with respect to the overextensions [5] nevertheless in [6] it is found numerically that this instability is stabilized at the nonlinear stage by the vertex splitting instability. Thus some controversy still remains with the experimental results shown in the review paper [7] where it was found a transformation of a labyrinthine pattern formed by the concentrated phase of a magnetic colloid into a hexagonal structure. In the present paper, analytical and numerical results are given which show that this transition can be explained by the peculiarities of the phase diagram of the magnetic colloid in the Hele-Shaw cell. They show that depending on the values of the physical parameters the different phases - the stripe phase, the hexagonal phase and the unmodulated phase can exist. Thus the transformation of the labyrinthine pattern into the hexagonal one in the frame of the present model is connected with a transition between those phases. That is illustrated here by the corresponding numerical simulation results.

\section{Phase diagram of the mag- netic colloid in the Hele-Shaw cell}

The phase diagram of a similar system - the polar amphiphile monolayer - has been calculated in $[8,9]$. From the point of view of our system that corresponds to the vanishing thickness of the Hele-Shaw cell. The calculation of the phase diagram for the finite thickness is carried out on the basis of the free energy proposed in [10]. It in dimensionless variables reads

$$
\begin{array}{r}
F=-\frac{1}{2} \int \phi^{2} d S+\frac{1}{4} \int \phi^{4} d S+\frac{1}{2} \int(\nabla \phi)^{2} d S+ \\
\frac{B m}{(h / l)^{2}} \int d S \int d S^{\prime} \phi(\vec{\rho}) \phi\left(\vec{\rho}^{\prime}\right)\left(\frac{1}{\sqrt{\left(\vec{\rho}-\vec{\rho}^{\prime}\right)^{2}}}-\frac{1}{\sqrt{\left(\vec{\rho}-\vec{\rho}^{\prime}\right)^{2}+(h / l)^{2}}}\right)
\end{array}
$$


where the following scales have been introduced $(\alpha=$ $\left.\frac{\partial^{2} M}{\partial n^{2}}\left(H-H_{c}\right), \gamma=\frac{1}{6} \frac{\partial^{3} \phi}{\partial n^{3}}\right)$ : length $-l=\sqrt{\beta / \alpha}$, the concentration $\delta n-\sqrt{\alpha / \gamma}$, the volume density of the free energy $\alpha^{2} / \gamma \cdot \vec{\rho}, \vec{\rho}^{\prime}$ - are the dimensionless radius-vectors of the points on the boundary of the Hele-Shaw cell, $h$ - a thickness of the cell, $\phi$ - a dimensionless concentration, the magnetic Bond number is defined according to the relation $\left(\frac{\partial M}{\partial n}\right)^{2} \frac{h}{l \alpha}$. The expression (1) for the free energy corresponds to the usual Landau expansion near the critical point of the demixing with account for the energy term due to the nonhomogenity of the concentration

$$
\frac{\beta}{2} \int(\nabla \delta n)^{2} d V
$$

and long-range interactions. Allowing the respective spatial modulation around $\phi$ for the concentration distribution $\phi$ in the stripe and the hexagonal phases

$$
\phi=\phi_{0}+\phi_{q} \cos q x
$$

and

$$
\phi=\phi_{0}+\sum_{j=1}^{3} A_{j} e^{i \overrightarrow{q_{j}} \vec{\rho}}+c . c .
$$

the respective free energies $f_{s}$ and $f_{h}$ of the two phases can be obtained $\left(A_{1}=A_{2}=A_{3}=A\right), \sum_{j=1}^{3} \vec{q}_{j}=0$. The results are

$$
\begin{aligned}
& f_{s}=-\frac{1}{2} \phi_{0}^{2}+\frac{1}{4} \phi_{0}^{4}+\frac{1}{3 \sqrt{3}} \frac{B m}{B m_{c}} \frac{h}{l} \phi_{0}^{2}+ \\
& +\frac{1}{4} \phi_{q}^{2}\left(\left(\frac{B m}{B m_{c}}\right)^{2 / 3}-1+3 \phi_{0}^{2}\right)+\frac{3}{32} \phi_{q}^{4}
\end{aligned}
$$

and

$$
\begin{array}{r}
f_{h}=-\frac{1}{2} \phi_{0}^{2}+\frac{1}{4} \phi_{0}^{4}+\frac{1}{3 \sqrt{3}} \frac{h}{l} \frac{B m}{B m_{c}} \phi_{0}^{2}+ \\
3 A^{2}\left(\left(\frac{B m}{B m_{c}}\right)^{2 / 3}-1+3 \phi_{0}^{2}\right)+12 \phi_{0} A^{3}+\frac{45}{2} A^{4}
\end{array}
$$

Here $B m_{c}=\frac{(h / l)^{2}}{3 \sqrt{3} \pi}$ is introduced. For $\frac{h}{l}$ large enough the wave number $q$ of the energetically optimal configuration is given by the relation

$$
q=\left(\frac{2 \pi B m}{(h / l)^{2}}\right)^{1 / 3}
$$

whatever the pattern. Minimizing the expression (2) and (3) with respect to $\phi_{q}$ and $A$ the dependencies of the free energies of the stripe and hexagonal phases on the average concentration in the Hele-Shaw cell can be found. The free energies calculated are shown in Fig. 1 and Fig. 2 for the stripe and the hexagonal phases correspondingly. Concerning Fig. 1 it is necessary to remark that the dependence is shown for $\mathrm{Bm}$ value less than the value $B m_{*}$ corresponding to the tricritical point of the transition between the stripe and unmodulated phases found in [11]. The value of $B m_{*}$ can be found from the equation

$$
3\left(\frac{B m_{*}}{B m_{c}}\right)^{2 / 3}=4-\frac{2}{3 \sqrt{3}} \frac{B m_{*}}{B m_{c}} \frac{h}{l}
$$

At $B m>B m_{*}$ transition between the unmodulated and the stripe phases is continuous. From Fig. 1 and Fig. 2 it is clear that a range of concentrations exists where, for the system, it is thermodynamically advantageous to phase separate into the two distinct phases - modulated and unmodulated. The concentrations of the phases at equilibrium are found from the double tangent construction

$$
\frac{\partial f_{i}}{\partial \phi_{0}}=\frac{\partial f}{\partial \phi_{0}}=\frac{f_{i}-f}{\phi_{i}-\phi}
$$

where $f_{i}, \phi_{i}(i=s, h)$ are respectively the values of the free energy and the concentrations of the modulated phases, and $f, \phi$ are the corresponding values for the unmodulated phase. It is important to remark that the actual equilibrium between the stripe and the unmodulated phases, in the frame of the present model, takes place at quite small $B m$ values. For values of $B m$ large enough in the intermediate concentration region, a stable hexagonal phase exists and the equilibrium between the stripe and and the hexagonal phases must be considered. The concentrations of those phases at equilibrium can be found from the construction similar to the one shown in Fig. 1 and Fig. 2. The resulting phase diagram is calculated and is shown in Fig. 3. It is then easy to see that, according to the present model and depending on the values of the physical parameters the system can find itself either in the stripe, the hexagonal or the unmodulated phases. Since the mean concentration $\phi_{0}$ is here associated to a distance from the colloidal critical point it depends on the magnetic field strength [12]. Then a variation of the external magnetic field allows to go from the region corresponding to one phase to the region corresponding to another one. Thus in the frame of the present model it is possible to give a quite natural interpretation to the observation in [7] of the transformation of the labyrinthine pattern to the hexagonal one. Namely due to the dependence of the critical concentration on the magnetic field strength a field increase shifts the system to the region of the phase space where the hexagonal phase is stable and as a result the transition from the stripe phase to the hexagonal one takes place. This possibility is illustrated by the results of the numerical simulation given in the next part. 


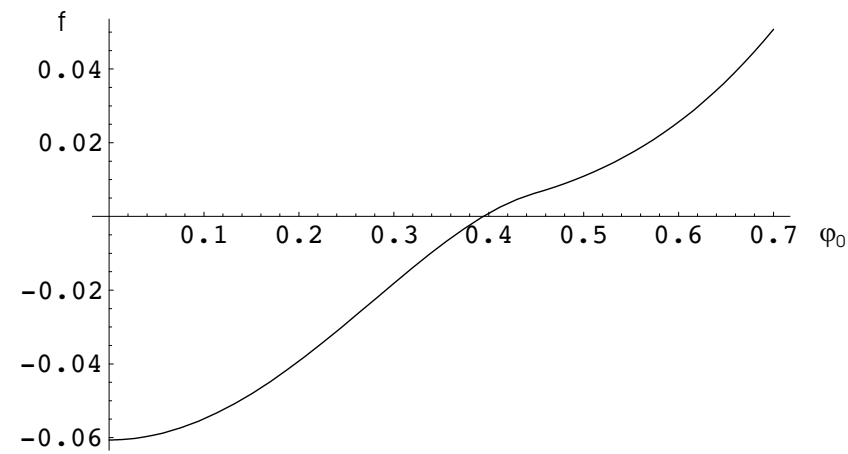

Figure 1. Free energy of the magnetic colloid below the tricritical point of the stripe-liquid transition. $\left(\frac{B m}{B m_{c}}=0.25<\right.$ $\left.\frac{B m_{*}}{B m_{c}} ; \frac{h}{l}=10\right)$.

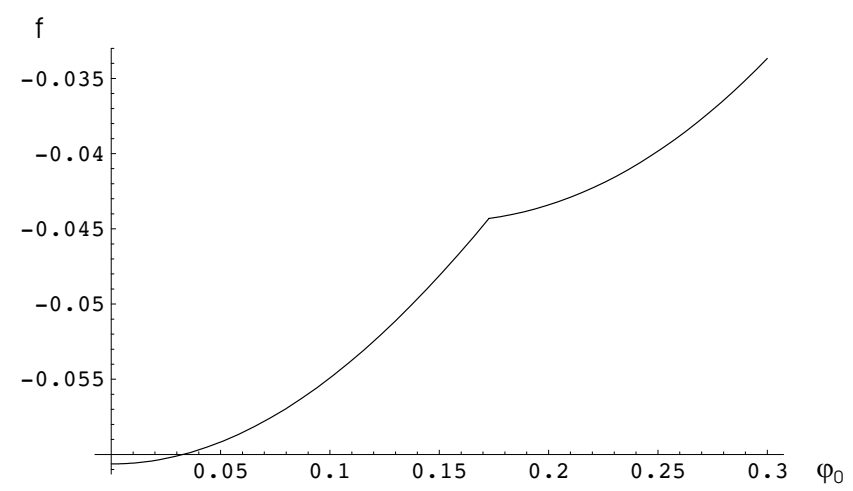

Figure 2. Free energies of the hexagonal and liquid phases $\left(\frac{B m}{B m_{c}}=0.25\right.$ and $\left.\frac{h}{l}=10\right)$. Concentrations of the phases in the equilibrium are found by the double tangent construction.

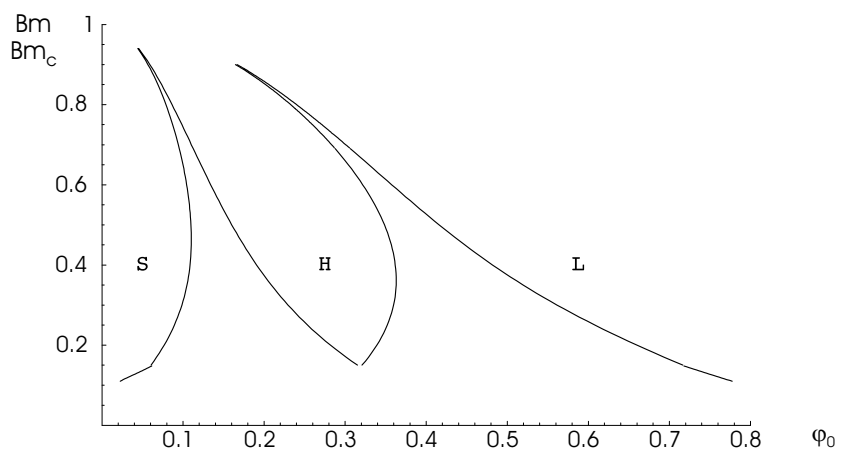

Figure 3. Phase diagram of the magnetic colloid in the HeleShaw cell. $\frac{h}{l}=10$.

\section{The results of numerical sim- ulation of the magnetic field induced phase transforma- tion}

The equation describing the concentration dynamics at the magnetic field induced phase transformation in a Hele-Shaw cell is derived in [13]. If put in a dimensionless form it reads

$$
\frac{\partial \phi}{\partial t}+\Delta\left(\phi-\phi^{3}+\Delta \phi-\frac{2 B m}{(h / l)^{2}} \int J\left(\vec{\rho}-\vec{\rho}^{\prime}, \frac{h}{l}\right) \phi\left(\vec{\rho}^{\prime}\right) d S^{\prime}\right)=0
$$

where $J(\vec{\rho}, h)=\frac{1}{\sqrt{\vec{\rho}^{2}}}-\frac{1}{\sqrt{\vec{\rho}^{2}+h^{2}}}$. The algorithm of the simulation of the concentration dynamics according to the equation (4) is based on the pseudospectral technique [13]. If the initial values of the physical parameters, associated to a labyrinth, are changed for those associated to a stable hexagonal pattern, then the transformation of the labyrinthine pattern to the hexagonal takes place. This is shown in Fig.4. It should be noted that the process shown in Fig. 4 is in good agreement with the phase diagram shown in Fig. 3 since at the values of the physical parameters chosen $\left(B m / B m_{c}=0.78, h / l=10, \phi_{0}=0.2\right)$ the sys- tem founds itself in the region of the phase space where the hexagonal phase is stable. The transitions between the hexagonal and the stripe phases are reversible. It is shown in Fig. 5 for the values of the physical parameters $\left(B m / B m_{c}=0.78, h / l=5, \phi_{0}=0.05\right)$ : the stripes form from the hexagonal structure initially obtained at $\left(B m / B m_{c}=0.78, h / l=5, \phi_{0}=0.2\right)$. Finally we can remark that if initial values of the physical parameters is chosen in the intermediate region between the stripe and the hexagonal phases, pattern containing a mixture of dumbbells and single droplets can be obtained, as it is shown in Fig. 6. 


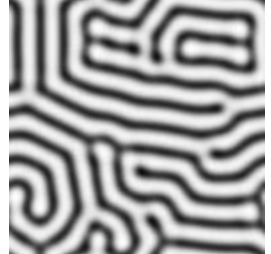

$t=3.1$

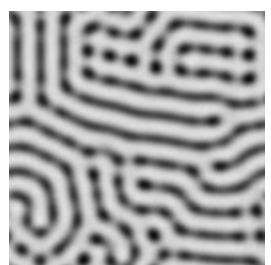

$\mathrm{t}=46.8$

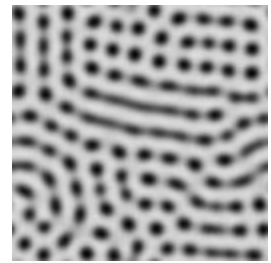

$t=93.6$

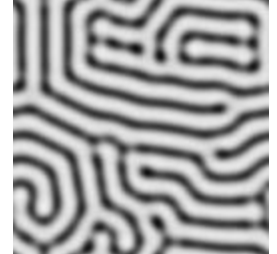

$=15.6$

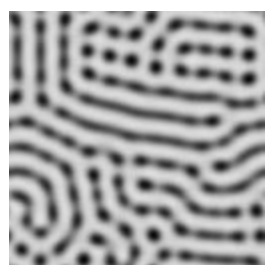

$\mathrm{t}=62.4$

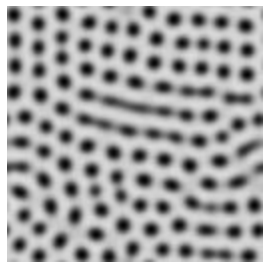

$t=124.8$

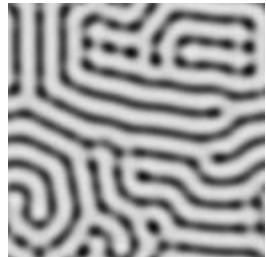

$t=31.2$

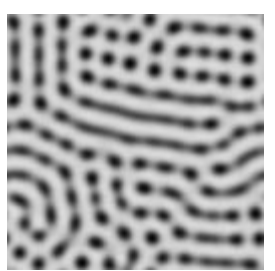

$\mathrm{t}=78.0$

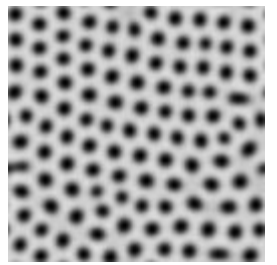

$t=249.6$

Figure 4. Transformation of the stripe structure to the hexagonal pattern $\left(\frac{B m}{B m_{c}}=0.78, \frac{h}{l}=10, \phi_{0}=0.2\right)$.

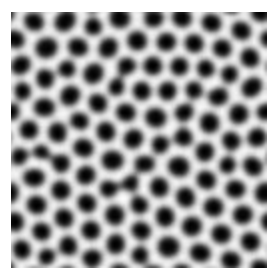

$t=73.4$

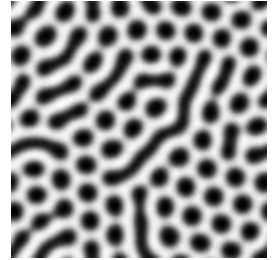

$t=514.0$

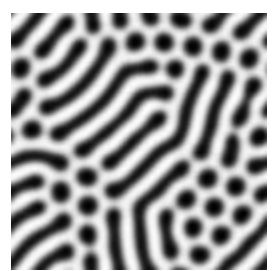

$t=1468.3$

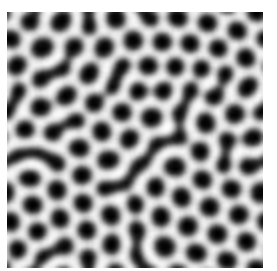

$t=220.4$

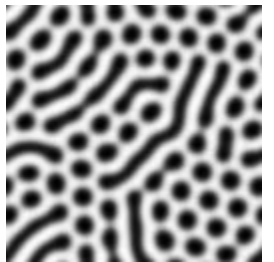

$t=734.7$

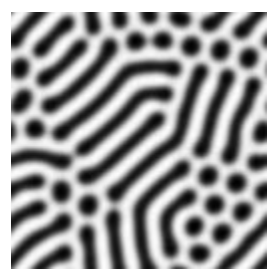

$t=3665.6$

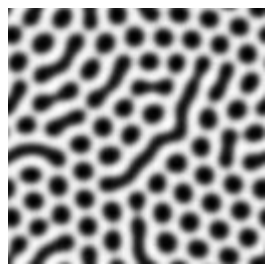

$t=367.2$

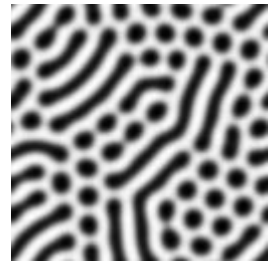

$t=1102.1$

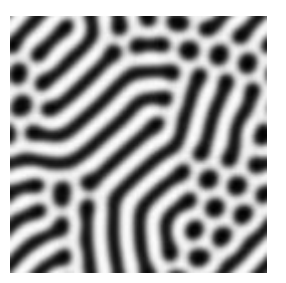

$t=5862.8$

Figure 5. Transformation of the hexagonal structure to the stripe structure at the decrease of the mean concentration. $\frac{B m}{B m_{c}}=0.78, \frac{h}{l}=5, \phi_{0}=0.05$. Initial pattern - the hexagonal structure formed at $\frac{B m}{B m_{c}}=0.78, \frac{h}{l}=5$ and $\phi_{0}=0.2$. 


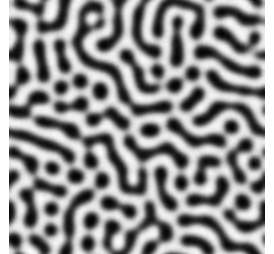

$t=76.7$

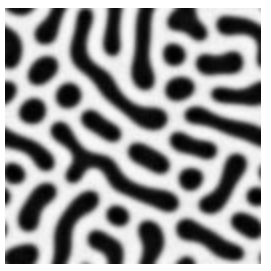

$t=766.8$

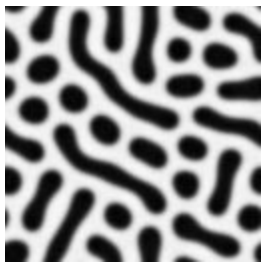

$\mathrm{t}=3841.9$

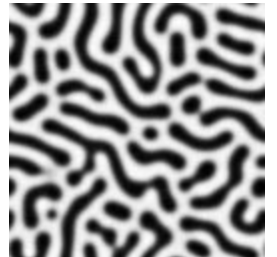

$t=153.4$

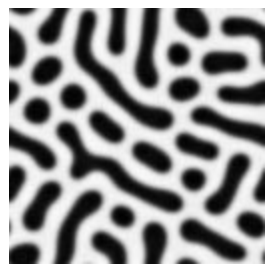

$t=1150.3$

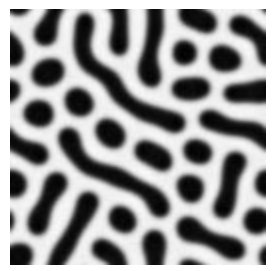

$\mathrm{t}=4602.8$

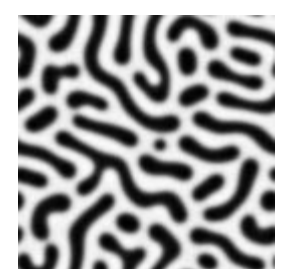

$\mathrm{t}=230.0$

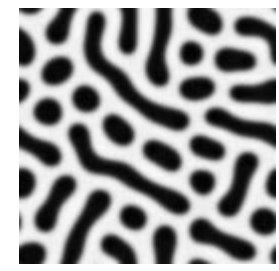

$t=2303.8$

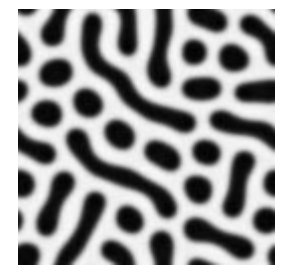

$t=6116.5$

Figure 6. Mixed intermediate state of the magnetic colloid $\left(\frac{B m}{B m_{c}}=0.3, \frac{h}{l}=10, \phi_{0}=0.075\right)$.

\section{Conclusion}

The calculated phase diagram of magnetic colloids in a Hele-Shaw cell shows the existence of stripe, hexagonal and unmodulated phases. The transitions between those phases can be caused by the variation of an external parameter: the magnetic field strength or the layer thickness. Those results allow to explain the experimental observation of the labyrinthine pattern transformation into an hexagonal structure of separated droplets by only the shifting the point in the phase space associated to the new magnetic field strength. An interesting issue about the character of the topological transformation taking place at the transformation of the stripe pattern to the structure of the separate droplets remains to be investigated.

\section{References}

[1] A. Cēbers, M.M. Mayorov. Structures of interface of a bubble and magnetic fluid in a field. Magnitnaya Gidrodinamika (in Russ.), 16, 231 (1980).

[2] Special issue Labyrinthine pattern formation. Magnitnaya Gidrodinamika, 35 (1999)

[3] R.E. Rosensweig, Ferrohydrodynamics Cambridge University Press, New York, 1985.

[4] F. Elias, C. Flament, J.C. Bacri, S. Neveu. Macro- organized patterns in ferrofluid layer: experimental studies. J. Physique I, 7 , 711 (1997).

[5] A. Cēbers, M.M. Mayorov. Magnetostatic instabilities in the plane layers of magnetizable fluids. Magnitnaya Gidrodinamika (in Russ.), 16, 27 (1980).

[6] A. Cēbers, I. Drikis. A numerical study of the evolution of quasi-two-dimensional magnetic fluid shapes. Magnitnaya Gidrodinamika, 32, 11 (1996).

[7] J.C. Bacri, R. Perzynski, D. Salin. Les liquides magnetiques. Recherche, 18, 1150 (1987).

[8] D. Andelman, Fr. Brochard, J.-F. Joanny. Phase transitions in Langmuir monolayers of polar molecules. Journal of Chemical Physics, 86, 3673 (1987).

[9] D. Andelman, Fr. Brochard, P.-G. de Gennes, J.F. Joanny. Transitions de monocouches a molecules polaires. C.R.Acad.Sc.Paris, 301, 675 (1985).

[10] A. Cēbers. On formation of periodical structures in flat layers by ferromagnetic colloids. Magnitnaya Gidrodinamika (in Russ.), 30, 132 (1986).

[11] A. Cēbers. Magnetic colloid pattern formation at magnetic field induced phase separation. Magnitnaya Gidrodinamika, 35344 (1999).

[12] A. Cēbers. Thermodynamic stability of magnetic liquids. Magnitnaya Gidrodinamika (in Russ.), 18, 42 (1982).

[13] A. Cēbers. Instabilities of concentration stripe patterns in ferrocolloids. Physical Review E, 61, 700 (2000). 